\title{
Commentary: Sortilin Inhibition Limits Secretion-induced Progranulin- dependent Breast Cancer Progression and Cancer Stem Cell Expansion
}

Sara Rhost ${ }^{1}$, Éamon Hughes' ${ }^{1}$, Anders Ståhlberg ${ }^{1,2,3}$, Göran Landberg ${ }^{1,2 *}$

'Department of Laboratory Medicine, Institute of Biomedicine, Sahlgrenska Cancer Center, University of Gothenburg, Gothenburg, Sweden ${ }^{2}$ Department of Clinical Pathology and Genetics, Sahlgrenska University Hospital, Gothenburg, Sweden

${ }^{3}$ Wallenberg Centre for Molecular and Translational Medicine, University of Gothenburg, Sweden

Article Info

\section{Article Notes}

Received: March 4, 2019

Accepted: March 26, 2019

\section{*Correspondence:}

Dr. Göran Landberg, Department of Laboratory Medicine Institute of Biomedicine, Sahlgrenska Cancer Center, University of Gothenburg, Gothenburg, Sweden; Email: goran.landberg@gu.se.

${ }^{\circledR} 2019$ Landberg G. This article is distributed under the terms of the Creative Commons Attribution 4.0 International License.
The genetically conserved glycoprotein progranulin is a pleiotropic factor that is implicated in wound healing, inflammation, neurodegeneration, lysosomal function and tumorigenesis among other biological events ${ }^{1,2}$. Progranulin is ubiquitously expressed and the fact that progranulin is genetically conserved and has a wide-ranging expression pattern indicates important biological functions. Progranulin acts as a holoprotein that can be cleaved into smaller granulin peptide domains by the activity of proteases, such as elastase and cathepsin $\mathrm{L}^{3}$. Cleavage of progranulin occurs in the extracellular matrix or within the lysosome. Interestingly, cleaved granulin peptides are described to display different biological functions compared to the precursor progranulin in wound healing, inflammation and neurodegeneration ${ }^{4,5}$. In breast cancer, high progranulin expression is associated with reduced disease-free and overall survival ${ }^{6,7}$ and is identified as a prognostic marker for ER $\alpha$ positive breast cancer ${ }^{8}$. Rhost and Hughes et al. recently reported that progranulin is differentially secreted by ER $\alpha$ positive and ER $\alpha$ negative breast cancer cell lines ${ }^{9}$. ER $\alpha$ negative cells showed high constitutive secretion of progranulin whereas the progranulin secretion fluctuated in ER $\alpha$ positive cells during microenvironmental stress, such as low oxygen. These results imply that subtypes of breast cancer regulate their progranulin secretion differently. Additional data obtained from Rhost et al. using ER $\alpha$ positive ductal carcinoma in situ (DCIS) samples with a hypoxic central area as an in vivo relevant model for hypoxia, revealed a clear gradient of progranulin expression towards the necrotic core (Figure 1). The increase of progranulin was further paralleled by an increase of HIF $1 \alpha$ and a decrease of ER $\alpha$ towards the inner edge of the DCIS lesions validating the association between progranulin and hypoxia as well as to ER $\alpha$. These data are in line with Lu et al. showing that overexpression of progranulin in ER $\alpha$ positive MCF7 cells induced estrogen independent proliferation via activation of cyclin D1 as well as tamoxifen resistance ${ }^{10}$. Collective data also support that hypoxia is not a solitary and local phenomenon but can actually influence distant biological processes including tumor progression and cancer treatment resistance. Growing evidence that the oxygen content of tumor tissue is an important determinant of metastasis ${ }^{11,12,13}$ further highlight the importance of the findings reported by Rhost and Hughes et al.

An interesting finding reported by Rhost and Hughes et al., is that recombinant progranulin and the granulin domain A peptide induced breast cancer stem cell propagation, both in vitro and in 
vivo, using ER $\alpha$ positive and ER $\alpha$ negative breast cancer cells. These results demonstrate that progranulin and granulin A indeed induce a more malignant form of breast cancer with a significantly increased capacity to form metastasis. The fact that granulin A mediates breast cancer stem cell spreading suggests that enzymatic cleavage of progranulin might play a role in rendering a more aggressive tumorigenesis. Although, previous results published by Sayers et al. describe contrasting results with an association of high secretion of Secretory Leukocyte Protease Inhibitor (SLPI) in highly metastatic cells ${ }^{14}$. SLPI nevertheless protects progranulin from enzymatic cleavage which indicates that inhibiting cleavage of progranulin
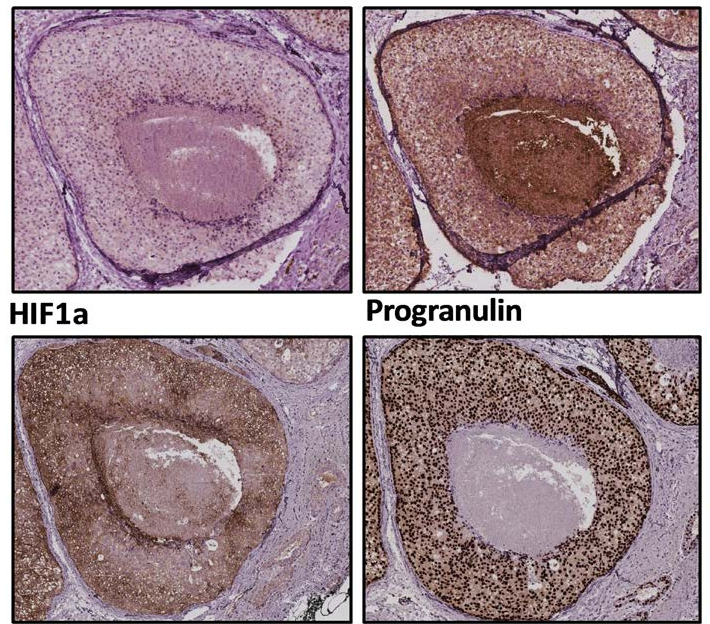

Progranulin

CA-9

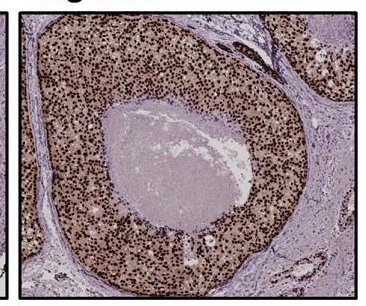

ERa

Figure 1: Immunohistochemical stainings of ductal carcinoma in situ (DCIS) samples. Immunohistochemical analyses of DCIS samples were performed using HIF1 $\alpha$, Progranulin, CA-9, ER $\alpha$ and Haematoxylin stainings. induce more metastatic cells. The general findings demonstrate that both progranulin and granulin peptides most likely are involved in tumor progression and future studies need to clarify the exact role for the granulins in the development of metastatic disease.

To improve our understanding of how progranulin and granulin induced breast cancer stem cell propagation, Rhost et al. further investigated the activation of several different kinases during progranulin and granulin treatment using a human phospho-kinase array. Data indicated that both progranulin and granulin A induced phosphorylation of several kinases, such as GSK3 $\alpha / \beta$ EGFR, MSK1/2, AMPKA1, AKT1/2/3, and mTOR, where progranulin was a more potent activator compared to granulin A (Figure 2). These results indicate that both progranulin and granulin A substantially influence several major intracellular signaling pathways. Interestingly, the PI3K/AKT/mTOR pathway is implicated in endocrine resistant $\mathrm{ER} \alpha$ positive breast cancer ${ }^{15}$ where it was shown that AKT activated the estrogen receptor pathway independently of estrogen availability. Further, mTOR inhibitors in combination with endocrine therapy was shown to overcome endocrine therapy resistance. The fact that progranulin induces AKT/ mTOR phosphorylation suggests a possible additional link between endocrine therapy resistance and progranulin via the AKT/mTOR pathway.

Detailed analyses at single-cell level further revealed that progranulin and its receptor sortilin were both associated with signature genes for differentiation and epithelial-mesenchymal transition (EMT) properties demonstrating that differentiated cancer cells respond to progranulin in a autocrine signalling pathway, whereby the sortilin (SORT1) positive subpopulation gradually shifted

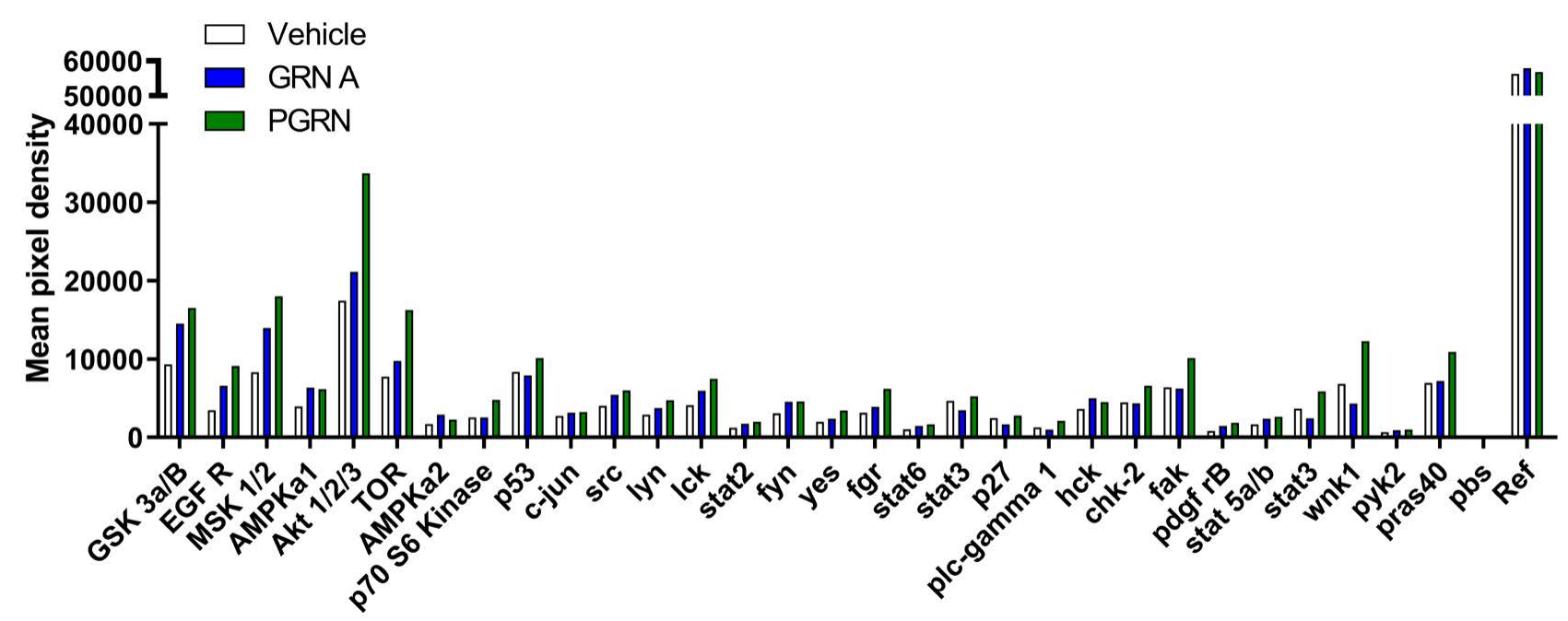

Figure 2: Progranulin and granulin A induce phospho-kinase expression in ER $\alpha$ positive MCF7 cells. MCF7 cells were treated with either vehicle (PBS) progranulin (PGRN, $1 \mu \mathrm{g} / \mathrm{ml}$ ) or granulin A (GRN A, $1 \mu \mathrm{g} / \mathrm{ml}$ ) for 50 minutes. Cell protein lysates $(\mathrm{n}: 2)$ were then analysed using a phospho-kinase array system (R\&D Systems). Data is expressed as mean pixel density. 
from highly differentiated cell types to more cancer stem cells-like cells ${ }^{9}$. Sortilin itself is reported to be associated with breast cancer aggressiveness ${ }^{16}$. Importantly, the spreading of breast cancer stem cells induced by granulin A could be blocked by the orally bioavailable small molecule AF38469 ${ }^{17}$, binding to sortilin. Further, elevated levels of progranulin or granulin A impacted breast cancer stem cells similarly through the receptor sortilin, despite subtype of breast cancer. Progranulin and granulin A induced breast cancer stem cell spreading resulted in a more aggressive cancer with a significant increase in metastasis formation. To further validate the findings clinically, we performed additional single-cell studies using primary breast cancer cells obtained from surgery to determine the relation between progranulin, sortilin and subpopulations defined by differentiation and cancer stem cell markers as earlier established $^{18}$. In total, 184 individual cells from an ER $\alpha$ positive breast cancer and corresponding axillary lymph node metastasis were analysed using principal component analysis. SORT1 and GRN clustered with the differentiation associated genes in the primary tumor (Figure $\mathbf{3 A}$ ) and upon metastatic spread, the expression of SORT1, GRN and a number of proliferative markers nevertheless clustered with cancer stem cell associated genes (Figure 3B). These results suggest that the SORT1 expressing population in the primary tumor was associated with highly differentiated cells, whereas SORT1 expressing cells during disease progression associated with genes related to cancer stem cell features.

Collective data concerning progranulin and sortilin presence in cancer cells and the now reported link to cancer stem cells and cancer progression could be of interest for future cancer therapy design with the potential to develop a unique cancer treatment based on selective targeting cancer stem cell propagation through sortilin. Sortilin is apart from being a receptor to progranulin also described to bind with high-affinity to the proinflammatory cytokines IL6 ${ }^{19}$. In breast cancer, IL6 is associated with increased tumor stage, lymph node infiltration, recurrence, and treatment resistance $\mathrm{e}^{20,21,22}$ further signifying the role of sortilin in breast cancer progression.

\section{Material and Methods}

\section{Single-cell qPCR analysis}

Single cell analyses were performed as earlier described ${ }^{22}$. The analyses of primary tumor samples were analysed according to the protocol described in Akrap et al. ${ }^{18}$.

\section{Human Phospho-Kinase Antibody Array}

The phospho-kinase antibody array was performed according to the protocol provided by the manufacturer (R\&D systems). Cell lysates from MCF7 cells treated with progranulin $(1 \mu \mathrm{g} / \mathrm{ml})$ or granulin A $(1 \mu \mathrm{g} / \mathrm{ml})$ for 50 minutes were analysed for phosphorylation of several different kinases using the human phospho-kinase antibody array.

\section{Immunohistochemistry}

Tissue sections were fixed with $4 \%$ phosphate buffered formaldehyde, embedded in paraffin and cut into $4.5 \mu \mathrm{m}$ thick sections. Immunohistochemistry was performed using DAKO Autostainer LINK 48 using Envision FLEX+ detection system. Briefly, deparaffinized sections were
A

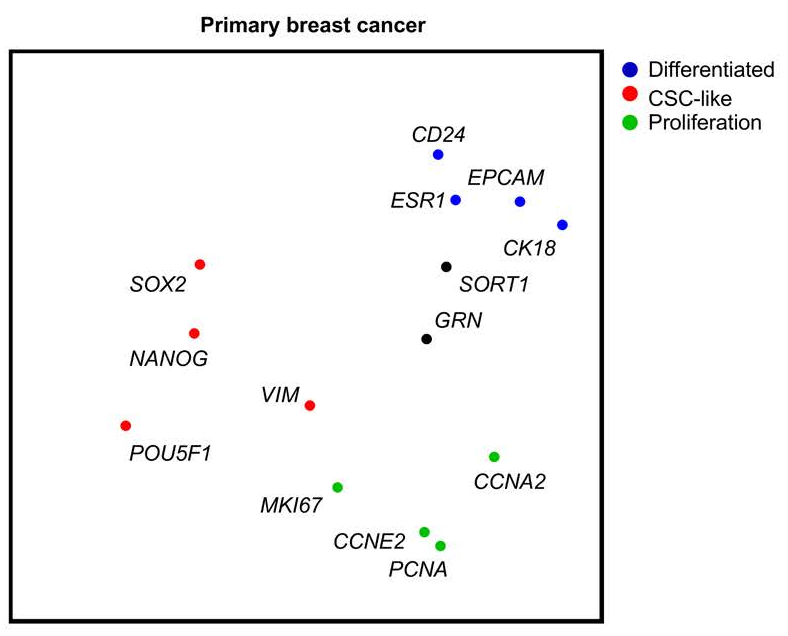

B

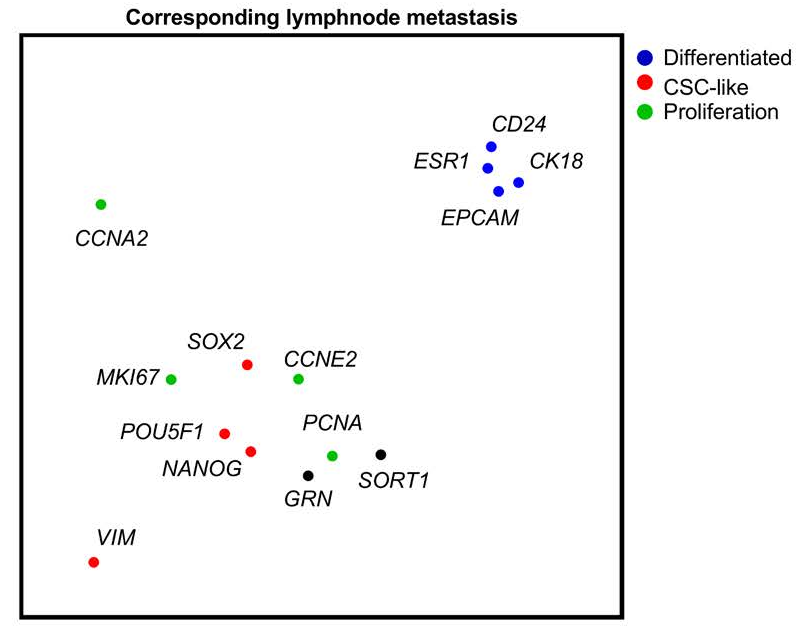

Figure 3: Differentiated sortilin positive cells change their transcriptional signature during disease progression in patient derived tumor samples. Single cell analyses of GRN and SORT1 (black) and sets of differentiation (blue), proliferation (green) and cancer stem cell -related (red) transcripts and the parallel gene cluster principal component analysis of (a) a primary ER $\alpha$ positive breast cancer and (b) the corresponding axillary lymph node metastases. 
subjected to antigen retrieval by high pressure cocking and DIVA antigen retrieval $\mathrm{pH} 6.2$, followed by blocking with $3 \%$ hydrogen peroxide and incubation with primary antibody against HIF1 $\alpha$ (ab82832, abcam), progranulin (MA1-187, Thermo scientific), CA-9 (ab15086, Abcam), ER $\alpha$ (M7047, Dako) at RT for 1 hour. For signal amplification EnVision $^{\text {TM }}$ FLEX+ rabbit linker (SM805, DAKO) and EnVision $^{\text {TM }}$ FLEX+ mouse linker (SM804, DAKO) was used, respectively. Further EnVision FLEX/HRP visualization reagent EnVision ${ }^{\mathrm{TM}}$ FLEX/HRP secondary antibody-coated polymer peroxidase complexes (\#SM802, DAKO), followed by DAB substrate/chromogen (DAKO) was used. Slides were counterstained with hematoxylin (DAKO). Stained sections were scanned by Leica SCN400 scanner at 20X and evaluated by the automated image analysis Definiens Developer XD tissue studio program.

\section{Acknowledgments}

We thank the patients from Sahlgrenska University Hospital who donated samples for this research and the pathology and surgery departments for patient consent and sample collection. We thank our colleagues Pernilla Gregersson, Karoline Berger, Daniel Andersson and Ylva Magnusson for their technical research contribution.

\section{Funding}

This work was supported by grants from Knut and Alice Wallenberg Foundation; Wallenberg Centre for Molecular and Translational Medicine, University of Gothenburg, Sweden; Swedish Cancer Society (2016-486 and 2016-438); Swedish Research Council (521-20125716, 16-06074 and 2017-01392); the Swedish state under the agreement between the Swedish government and the county councils; the ALF-agreement (7211091 and 716321); VINNOVA; Assar Gabrielssons Research Foundation; BioCARE National Strategic Research Program at University of Gothenburg; Johan Jansson Foundation for Cancer Research; Wilhelm and Martina Lundgren Foundation for Scientific Research.

\section{References}

1. De Muynck L, Van Damme P. Cellular effects of progranulin in health and disease. J Mol Neurosci. 2011; 45(3): 549-60.

2. Kao AW, McKay A, Singh PP, et al. Progranulin, lysosomal regulation and neurodegenerative disease. Nat Rev Neurosci. 2017; 18(6): 325-333.

3. Lee CW, Stankowski JN, Chew J, et al. The lysosomal protein cathepsin L is a progranulin protease. Mol Neurodegener. 2017; 12(1): 55.

4. Shoyab M, McDonald VL, Byles C, et al. Epithelins 1 and 2: isolation and characterization of two cysteine-rich growth-modulating proteins. Proc Natl Acad Sci U S A. 1990; 87(20): 7912-6.

5. Tolkatchev D, Malik S, Vinogradova A, Wang P, et al. Structure dissection of human progranulin identifies well-folded granulin/ epithelin modules with unique functional activities. Protein Sci. 2008; 17(4): 711-24.
6. Tkaczuk KR, Yue B, Zhan M, et al. Increased Circulating Level of the Survival Factor GP88 (Progranulin) in the Serum of Breast Cancer Patients When Compared to Healthy Subjects. Breast Cancer (Auckl). 2011; 5: 155-62.

7. Serrero G, Hawkins DM, Yue B, et al. Progranulin (GP88) tumor tissue expression is associated with increased risk of recurrence in breast cancer patients diagnosed with estrogen receptor positive invasive ductal carcinoma. Breast Cancer Res. 2012; 14(1): R26.

8. Koo DH, Park CY, Lee ES, et al. Progranulin as a prognostic biomarker for breast cancer recurrence in patients who had hormone receptorpositive tumors: a cohort study. PLoS One. 2012; 7(6): e39880.

9. Rhost S, Hughes É, Harrison $\mathrm{H}$, et al. Sortilin inhibition limits secretion-induced progranulin-dependent breast cancer progression and cancer stem cell expansion. Breast Cancer Res. 2018; 20(1): 137.

10. Lu R, Serrero G. Mediation of estrogen mitogenic effect in human breast cancer MCF-7 cells by PC-cell-derived growth factor (PCDGF/ granulin precursor). Proc Natl Acad Sci U S A. 2001; 98(1): 142-7.

11. Bellot G, Garcia-Medina R, Gounon P, et al. Hypoxia-induced autophagy is mediated through hypoxia-inducible factor induction of BNIP3 and BNIP3L via their BH3 domains. Mol Cell Biol. 2009; 29(10): 2570-81.

12. Bayer C, Vaupel P. Acute versus chronic hypoxia in tumors: Controversial data concerning time frames and biological consequences. Strahlenther Onkol. 2012; 188(7): 616-27.

13. Rofstad EK, Gaustad JV, Egeland TA, et al. Tumors exposed to acute cyclic hypoxic stress show enhanced angiogenesis, perfusion and metastatic dissemination. Int J Cancer. 2010; 127(7): 1535-46.

14. Sayers KT, Brooks AD, Sayers TJ, et al. Increased secretory leukocyte protease inhibitor (SLPI) production by highly metastatic mouse breast cancer cells. PLoS One. 2014; 9(8): e104223.

15. Paplomata E, O'Regan R. New and emerging treatments for estrogen receptor-positive breast cancer: focus on everolimus. Ther Clin Risk Manag. 2013; 9: 27-36.

16. Roselli S, Pundavela J, Demont Y, et al. Sortilin is associated with breast cancer aggressiveness and contributes to tumor cell adhesion and invasion. Oncotarget. 2015; 6(12): 10473-86.

17. Schrøder TJ, Christensen S, Lindberg $S$, et al. The identification of AF38469: an orally bioavailable inhibitor of the VPS10P family sorting receptor Sortilin. Bioorg Med Chem Lett. 2014; 24(1): 177-80.

18. Akrap N, Andersson D, Bom E, et al. Identification of Distinct Breast Cancer Stem Cell Populations Based on Single-Cell Analyses of Functionally Enriched Stem and Progenitor Pools. Stem Cell Reports. 2016; 6(1): 121-36.

19. Mortensen MB, Kjolby M, Gunnersen S, et al. Targeting sortilin in immune cells reduces proinflammatory cytokines and atherosclerosis. J Clin Invest. 2014; 124(12): 5317-22.

20. Knupfer H, Preiss R. Significance of interleukin-6 (IL-6) in breast cancer (review). Breast Cancer Res Treat. 2007; 102(2): 129-35.

21. Salgado R, Junius S, Benoy I, et al. Circulating interleukin-6 predicts survival in patients with metastatic breast cancer. Int J Cancer. 2003; 103(5): 642-6.

22. Zhang GJ, Adachi I. Serum interleukin-6 levels correlate to tumor progression and prognosis in metastatic breast carcinoma. Anticancer Res. 1999; 19(2B): 1427-32.

23. Ståhlberg A, Rusnakova V, Kubista M. The added value of single-cell gene expression profiling. Briefings in Functional Genomics. 2013; 12(2): 81-89. 